Article

\title{
Residual Stress of a TC17 Titanium Alloy after Belt Grinding and Its Impact on the Fatigue Life
}

\author{
Yi He, Guijian Xiao *, Wei Li and Yun Huang
}

The State Key Laboratory of Mechanical Transmissions, Chongqing University, Chongqing 400044, China; m18423397236@163.com (Y.H.); 13452455686@163.com (W.L.); yunhuang@samhida.com (Y.H.)

* Correspondence: xiaoguijian@cqu.edu.cn; Tel.: +86-023-6766-9883

Received: 12 October 2018; Accepted: 6 November 2018; Published: 8 November 2018

\begin{abstract}
Titanium alloy materials are widely used in the design of key parts, such as aeroengine blades and integral blades. The surface residual stress has a great influence on the fatigue life of the parts mentioned above. Presently, abrasive belt grinding can form residual stress on the surface. However, the formation mechanism has not yet been revealed, providing the impetus for the present study. First of all, the surface residual stress is characterized based on Bragg's law. The influence of contact force, reciprocating frequency, and feed speed on the residual stress of a titanium alloy abrasive belt grinding is obtained using an experimental method. The residual stress model is simulated by the tensile force on the surface of the model, and the fatigue life of the bar under a sinusoidal tensile load is analyzed by simulating the fatigue test of the titanium alloy bar. Finally, fatigue testing and fracture analysis are carried out. The experimental results show that with the increase of the grinding contact force, increase of the reciprocating frequency, and decrease of the feed speed, the residual compressive stress on the surface of the parts increases and the fatigue life is higher at the same working stress level. It also shows that the residual compressive stress produced by abrasive belt grinding is in the range of 120-300 MPa. The fatigue simulation curve's inflection point appears at the level of $550 \mathrm{MPa}$. The error between the simulation data and the experimental data is less than $10 \%$, which shows the accuracy of the simulation experiment. The fracture morphology at room temperature is a ductile fracture with fine equiaxed dimples.
\end{abstract}

Keywords: TC17 titanium alloy; belt grinding; residual stress; fatigue failure

\section{Introduction}

Titanium alloys were developed in the middle of the 20th century and are key metal materials, because titanium has a lower density, better corrosion resistance, and higher strength than many other metals. They are widely used in the design and manufacture of aeroengine compressor blades, integral blades, and fan blades, because of their high heat resistance and other excellent properties [1]. China's Shenzhou series spacecraft and the recent, independently developed domestic passenger plane C919 indicate that China has become a significantly space-focused country, and the amount of titanium alloys used in the aerospace and other manufacturing fields is increasing year by year, with alloys playing an increasingly important role. It has become an important basis for judging the advanced nature of major aerospace parts and equipment; the service life of these key parts made from titanium alloys is closely related to the service life and quality of these important achievements, and the most common failure form is fatigue failure [2] (low cycle fatigue and high cycle fatigue) under service conditions. Therefore, it is of great practical significance to analyze the fatigue life of titanium alloys.

As is well-known, the reasonable distribution of residual stress on mechanical parts is of great significance to improve its performance. It is especially suitable for the components bearing an alternate load [3]. Bhaumik et al. [4] found that about $60 \%$ of the in-service faults of aerospace 
components are caused by fatigue, and the surface integrity characteristics, such as residual stress, have a great influence on the fatigue life of aeronautic and spaceflight components. It is very important to explore the influence law guiding engineering practice. However, the mechanism of the influence of abrasive belt grinding on the fatigue life of titanium alloys has not been grasped presently. Therefore, it is difficult to guide the formulation of the evaluation standard of surface characteristics for the optimization of the fatigue life of titanium alloys.

In their research on surface integrity in titanium alloy grinding, Bigerelle et al. [5,6] proposed a method to characterize surface roughness in the process of tool wear, and a series of roughness process parameters were used to characterize the surface integrity, processing dynamics, and mechanical properties. In addition, the fractal model of the wearing process in polishing and the model of the machining process with polishing were established. Jourani et al. [7] studied the relationship between the abrasive belt structure and surface quality, and analyzed the physical mechanism of abrasive belt grinding and polishing. The mathematical model of the process of material removal on the surface of the parts was established. Segreto [8] applied the traditional extraction method based on statistics, and the feature extraction method based on wavelet packet transform to the sensor signal detected in the polishing process. The surface roughness level of polished parts was determined. Eriksen et al. [9] combined the traditional tool manufacturing process and a new robot-assisted polishing method to form multifunctional surfaces.

Xiao et al. [10] analyzed the influence of the abrasive belt size and rough grinding pressure on the grinding surface roughness, work hardening, metallographic structure, and residual stress of a titanium alloy blade's abrasive belt using experiments, and established a regression model of the surface roughness. Zhao [11] studied the prediction of the blade surface roughness and the model of parameter optimization in the polishing of flexible abrasive tools. The model obtained by the experiments could improve the surface roughness by $25 \%$. Xiao et al. [12,13] studied the adaptive belt grinding method for the edge of aeronautical blades, and analyzed the surface roughness after belt grinding. The results showed that after belt grinding, the surface roughness is less than $0.25 \mu \mathrm{m}$, and the surface is under compressive stress. Dong et al. [14] ground a titanium alloy by using two kinds of microcrystalline corundum grinding wheels with large porosity and common porosity, respectively. The experimental results show that the large-porosity grinding wheel could obtain a better surface quality.

The research on surface integrity and fatigue life includes fatigue failure types, such as high and low cycle fatigue, bending fatigue, rolling contact fatigue, and so on.

Jeelani and Ramakrishnan [15] studied the machined surface integrity and fatigue life of a Ti-6Al-2Sn-4Zr-2Mo titanium alloy. The results showed that there were many defects on the machined surface. However, the surface integrity quality of titanium alloys can be improved, and thus the service life of the part can be improved. This is due to the formation of residual compressive stress on the surface of the part, which can reach $275 \mathrm{MPa}$. The surface integrity and fatigue life of a machined Ti-Al alloy were also studied by Mantle et al. [16] The results show that the microcracks (with a depth less than $20 \mu \mathrm{m}$ ) produced in the turning process of the titanium alloy can reduce the life of the parts. In recent years, Lee et al. [17] and Bhaumil et al. [18] calculated the effect of roughness on the contact fatigue using mesoscopic methods. The results showed that the fatigue properties of the materials with a roughness greater than $0.4 \mu \mathrm{m}$ will be significantly affected. James et al. [19] found that when the average stress is lower than the yield point of $20 \mathrm{MPa}$, there is a linear relationship between residual stress and the number of experimental cycles to fatigue.

Huang et al. [20] studied the effect of different surface integrity qualities on the low cycle and high cycle fatigue life of a GH33A superalloy at high and normal temperatures, which provided the experimental data for the design of a new engine turbine disk. Sun and Huang [21] studied the effect of different surface working conditions on the fatigue life of a high-strength titanium alloy. The results showed that the fatigue strength of the high-strength titanium alloy increased by about one third when a surface compressive stress layer of $250 \mu \mathrm{m}$ was introduced. At the same time, the fatigue resistance 
was increased by about $70 \%$ when the surface was smooth and flat without defects. Li Kang et al. [22] modified the surface of a TC4 titanium alloy using wet shot peening. The fatigue crack initiation position of the specimen was transferred from the surface to the region, $1 \mathrm{~mm}$ deep inside the specimen, thus the fatigue life of the material was improved significantly. Wang Xin et al. [23] used ceramic shot peening to treat the ground surface of an alloy. The results show that the fatigue limit of alloy grinding decreased from $583 \mathrm{MPa}$ to $465 \mathrm{MPa}$ at $650^{\circ} \mathrm{C}$ when the stress concentration factor was increased from $\mathrm{Kt}=1$ to $\mathrm{Kt}=1.7$. After high-strength shot peening, the fatigue limit of $\mathrm{Kt}=1.7$ returned to $530 \mathrm{MPa}$, and the low-strength shot peening had no effect on the fatigue limit of $\mathrm{Kt}=1.7$. Zhou [24] applied the research results of a laser impact composite strengthening mechanism to the turbine blade of a certain aeroengine, and focused on the application of turbine blade tenon. The fatigue life of the turbine blade at high and low temperatures was improved by $379 \%$.

From the above, it can be seen that the research on the influence of the surface integrity and of the surface integrity on the fatigue life of titanium alloy abrasive belt grinding has been focused on the surface texture optimization, such as the working environment temperature, surface roughness, and so on. However, there is little research on residual stress on the surface of titanium alloy grinding, so it is difficult to understand the mechanism of the residual stress on the fatigue life of the grinding surface of the titanium alloy abrasive belt. The residual stress of the TC17 titanium alloy after belt grinding and its impact on fatigue life is presented here. In this study, we reveal the influence of the three main grinding parameters on residual stress, establish the characterization model of residual stress, predict the fatigue life of the TC17 bar after grinding by simulation, and carry out a fatigue experiment. The influence of various parameters on fatigue life is studied, and the experimental fracture surface is analyzed and discussed, which provides a reference for the influence of the fatigue life of titanium alloys after grinding with an abrasive belt.

\section{Materials and Methods}

\subsection{Materials}

The chemical composition and physical properties of the TC17 material are shown in Tables 1 and 2, as follows:

Table 1. Material composition.

\begin{tabular}{lcccccc}
\hline Component & $\mathrm{Al}$ & $\mathrm{Sn}$ & $\mathrm{Zr}$ & $\mathrm{Mo}$ & $\mathrm{Gr}$ & $\mathrm{Ti}$ \\
\hline Content (\%) & $5 \%$ & $2 \%$ & $2 \%$ & $4 \%$ & $4 \%$ & balance \\
\hline
\end{tabular}

Table 2. Material physical properties.

\begin{tabular}{cccccc}
\hline Elastic Modulus (GPa) & Elongation (\%) & Shrinkage (\%) & Density $\left(\mathbf{k g} / \mathbf{m}^{3}\right)$ & Yield Strength (MPa) & Tensile Strength (MPa) \\
\hline 111.5 & 10 & 17.5 & 4640 & 1110 & 1180 \\
\hline
\end{tabular}

The TC17 alloy is a kind of $\alpha / \beta$-type hot strong titanium alloy with excellent comprehensive properties and is rich in the $\beta$-phase, which has a high strength, good fracture toughness, large quenching depth, wide stable range of forging, low thermal conductivity, and high cutting temperature. However, it also has some disadvantages, such as a low elastic modulus, easy deformation, high grinding force, serious tool wear, and cold hardening $[25,26]$.

\subsection{Methods}

The surface of a TC17 alloy bar was machined with different abrasive belt grinding process parameters, and the residual stress values of the titanium alloy surface under different technological parameters were measured and were compared with each other. Finally, the conclusion was drawn.

The experimental device is shown in Figure 1, as follows: Figure 1a is a grinding device with titanium alloy rods, which is composed of a belt (rough grinding with $\mathrm{Al}_{2} \mathrm{O}_{3}$ abrasive grain, $50 \mu \mathrm{m}$ 
and finish grinding with SC abrasive grain, $20 \mu \mathrm{m}$ ), force control shaft, cooling jet, contract wheel, adaptive clamp, TC17 bar, and belt grinding system. The two ends of the TC17 bar are rotated by the adaptive clamp to finish the circumferential grinding. At the same time, the axial grinding of the force control shaft is carried out along the axial motion. Figure $1 \mathrm{~b}$ is the belt grinding system. In Figure $1 \mathrm{~b}$, $F_{\mathrm{a}}$ is the force of the belt on the workpiece, $v_{\mathrm{p}}$ is the feed speed, and $v_{\mathrm{s}}$ is the grinding speed. Figure $1 \mathrm{c}$ shows the distribution of the five test points where the residual stress of each workpiece was measured. Each group consisted of three workpieces.
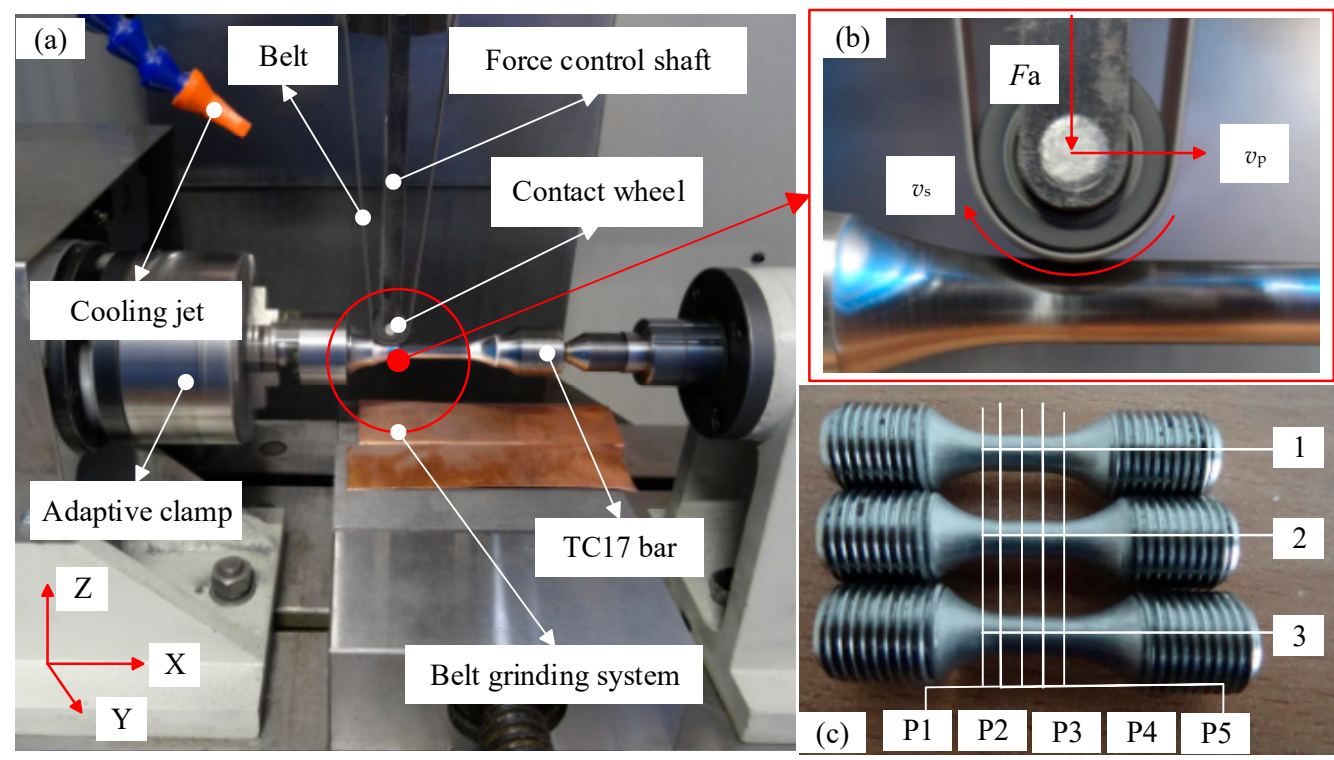

Figure 1. Experimental device and experimental materials: (a) grinding device with titanium alloy rods; (b) belt grinding system; (c) distribution of the five test points.

\section{Analysis of Surface Residual Stress Characteristics}

\subsection{Characterization of Surface Residual Stress Based on Titanium Alloy Belt Grinding}

In the grinding process, the abrasive is extruded with the workpiece under the normal grinding pressure, and is fed along the axial direction at the same time, as shown in Figure 2. In this process, because of the grinding heat and the direction of the abrasive feed extrusion, the stresses $\sigma_{1}$ and $\sigma_{2}$ are produced by the grinding heat and extrusion, respectively, and appear as a residual tensile stress state. The residual compressive stress produced when the grinding force $F_{\mathrm{a}}$ is combined with these two stresses to obtain the final surface residual stress distribution. When the residual compressive stress produced by $F_{\mathrm{a}}$ is greater than $\sigma_{1}$ and $\sigma_{2}$, the surface presents a state of compressive stress. Otherwise, there will be a residual tensile stress state. This is why the residual stress distribution in different states can be obtained by controlling the grinding pressure in abrasive belt grinding.

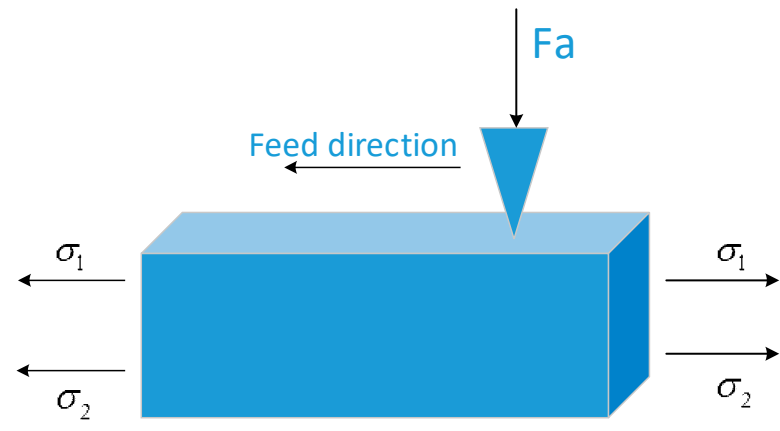

Figure 2. Stress load during single abrasive grinding. 
For titanium alloy polycrystalline materials, the corresponding macroscopic strain of residual stress is considered to be the sum of the lattice strain. Therefore, residual stress can be calculated by measuring the lattice strain, based on Hooke's law and the elastic mechanics theory. The magnitude of residual stress can be calculated using the X-ray diffraction method based on Bragg's law.

$$
n \cdot \lambda=2 d \cdot \sin \theta,
$$

where $n$ is the integer representing the diffraction order, $\lambda$ is the wavelength of the X-ray, $d$ is the crystal plane spacing of the crystal plane, and $\theta$ is the diffraction angle. When the contact force, $F$, and stresses, $\sigma_{1}$ and $\sigma_{2}$, cause the lattice spacing, $d$, to change, the diffraction angle, $\theta$, changes, so the change of $d$ can be obtained by measuring $\theta$, and the magnitude of stress in a certain direction can be obtained according to the elastic mechanics formula, as follows:

$$
\sigma_{x}=K \cdot M,
$$

where $K$ is the stress constant and $M$ is measured experimentally.

$$
\begin{gathered}
K=-\frac{E}{2(1+\mu)} \cdot \operatorname{ctg} \theta_{0}, \\
M=\frac{\partial(2 \theta)}{\partial\left(\sin ^{2} \psi\right)} .
\end{gathered}
$$

In the above equations:

$K$-Stress constant;

E-Elastic modulus of the materials;

$\mu$-Poisson's ratio of the measured crystal planes of materials;

$\theta_{0}$-Diffraction angles of materials without stress;

$M$-Slope of $2 \theta$ to $\sin ^{2} \psi$;

$\psi$-Angle between the normal direction of the crystal diffraction plane and the normal line of the sample surface [27].

For the characterization of residual stress, the forms of residual stress are different according to the different service environments of the different components. For example, the residual stress induced by the shot peening of the mechanical parts is affected by many technological parameters, including the type, size, hardness, and velocity of the projectile injection, in addition to being related to the material of the shot-peening part. The distance between the nozzle and spray surface, injection angle, shot-peening time, and coverage rate are mutually restricted. In order to measure the comprehensive effect of these parameters on residual stress, the magnitude of residual stress is usually characterized by the shot-peening strength. In the study of the rolling contact properties of materials, the influence of the gradient, magnitude, and depth of residual stress on the rolling contact properties is usually selected.

The finite element method is a very popular method that has been widely used in many fields [28-30]; thus, we have used this method in the current paper. In the simulation experiment, normal stress of a different magnitude is applied on the outer surface of the bar to characterize the residual stress of a different magnitude. When the stress is applied on the surface, the stress's effect on the element is as shown in Figure 3a. The distribution of the residual stress on the surface of the material is obtained by applying the normal tensile stress and the compressive stress to the surface of the material, respectively. The stress and deformation of the hexahedron mesh on the surface of the bar, subjected to tensile force, and the situation after the tensile stress loading can be seen in Figure $3 \mathrm{~b}$. We can clearly see that the total deformation of the hexahedron is reduced (dL1 > dL2 and dB1 > dB2) because of the stress of the upper and lower surfaces, the damage of the tensile work is reduced, and the number of fatigue cycles is increased. So, the residual compressive stress is simulated using tensile stress in this simulation experiment. Figure $3 \mathrm{c}$ shows the model with normal stress on the 
middle surface in the ANSYS Workbench software (ANSYS Workench 14.5, ANSYS, Canonsburg, PA, USA).

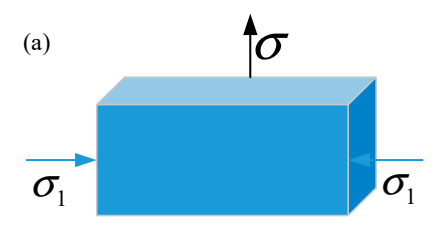

(b)

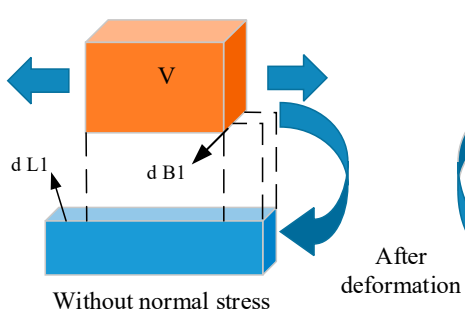

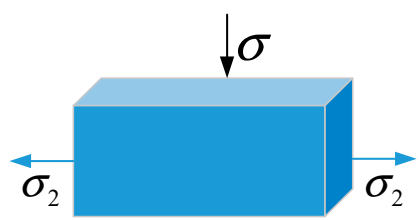

(c)

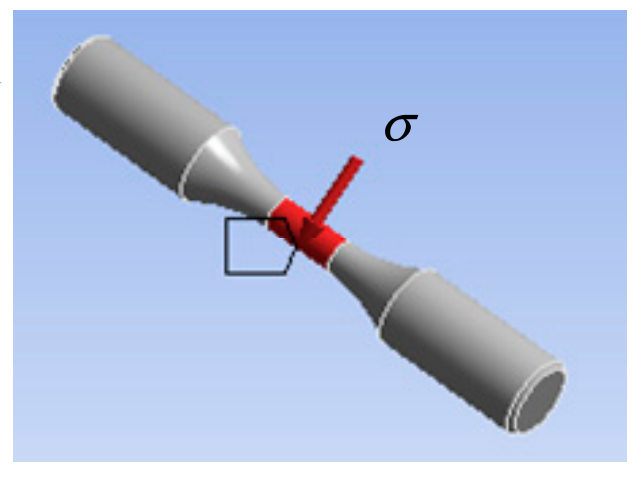

Figure 3. Residual stress simulation: (a) stress's effect on the element; (b) deformation of the element; (c) model with normal stress on the middle surface.

\subsection{Influence of Grinding Parameters on Surface Residual Stress}

The Panalytical X-ray projector was used to measure the surface residual stress after belt polishing, and the repetitive positioning accuracy of the goniometer and minimum step was 0.0001 degrees.

The thickness of the residual stress layer measured by the X-ray projector is shown in Figure 4. When the surface depth is less than $1.2 \mu \mathrm{m}$, the residual compressive stress increases with the increase of the surface depth, and decreases with the decrease of the surface depth.

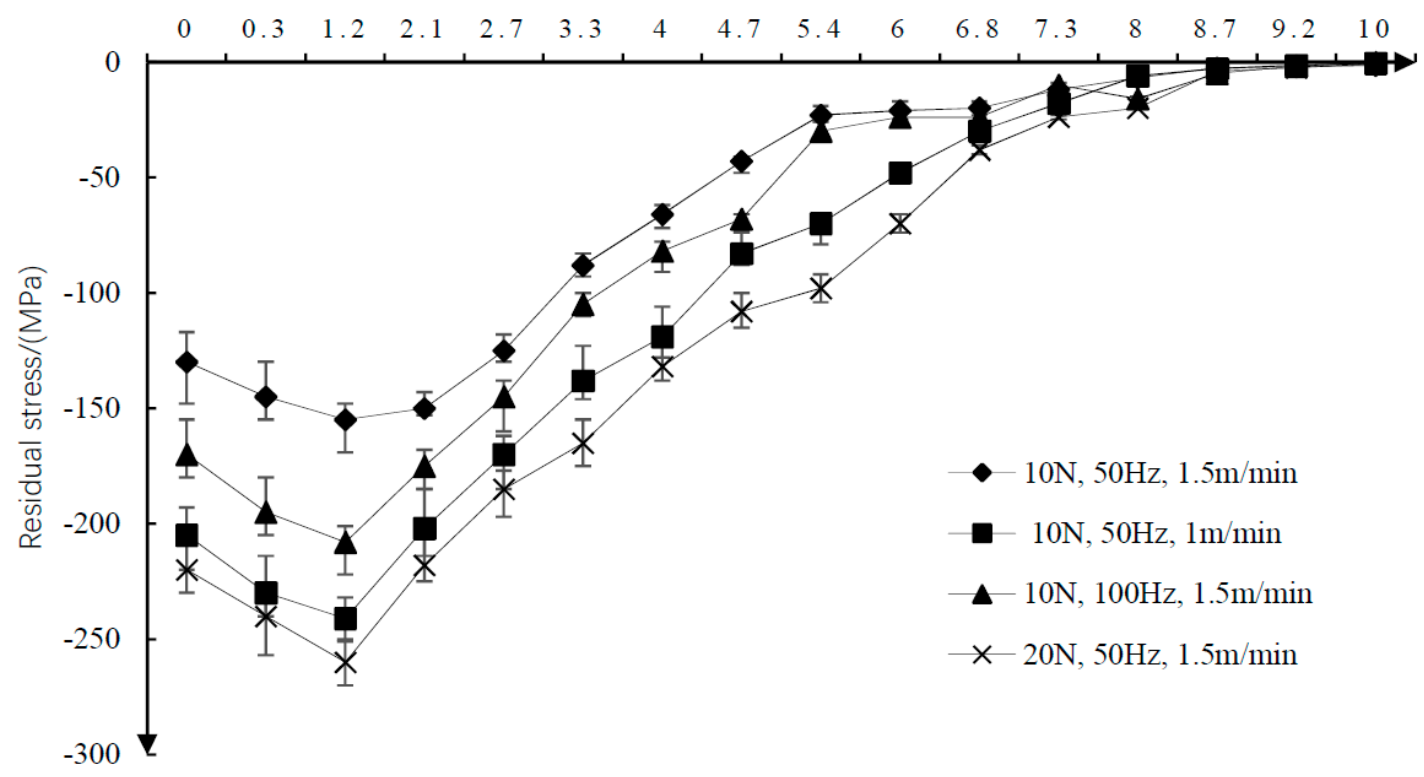

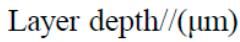

Figure 4. Layer depth of residual stress.

As shown in Figure 5, when the contact force is $10 \mathrm{~N}$, the average residual stress is $-106 \mathrm{MPa}$. When the contact force is increased to $30 \mathrm{~N}$, the average residual stress is $-311 \mathrm{MPa}$. Therefore, the residual compressive stress increases with the increase of the contact force. At the same time, with the increase of the contact force, the consistency of the residual compressive stress is improved. 


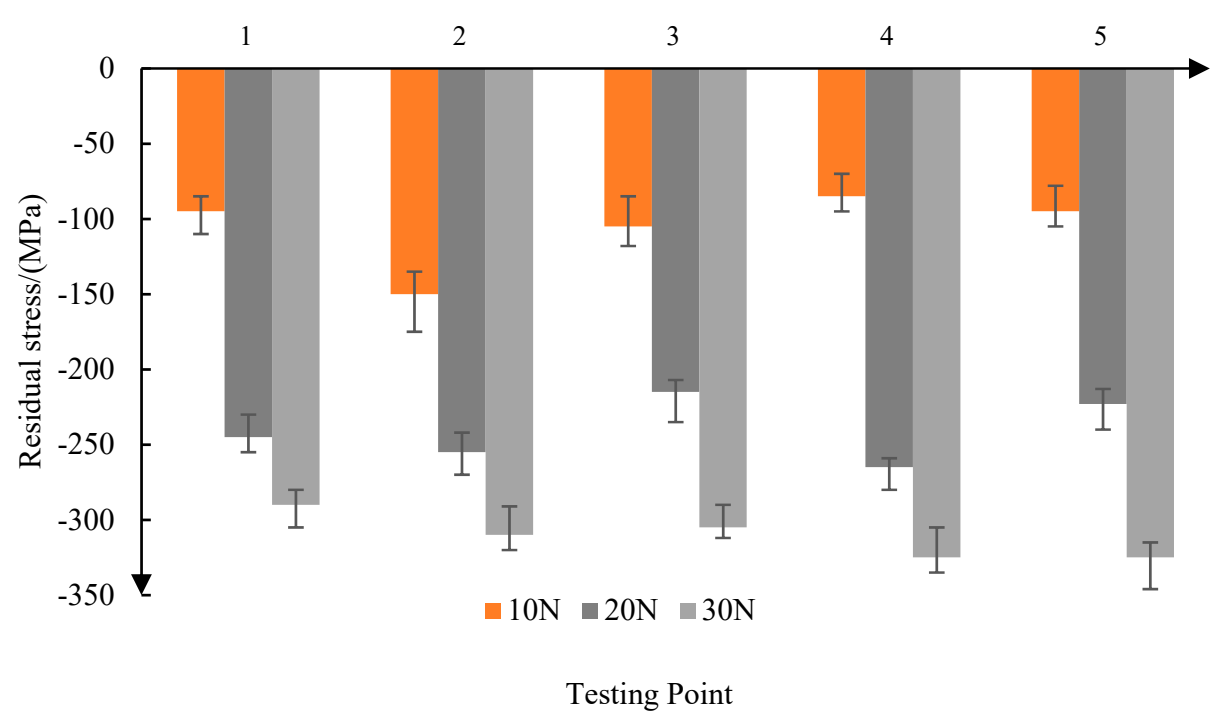

Figure 5. Influence of contact force on residual stress.

As shown in Figure 6, when the reciprocating frequency is $50 \mathrm{~Hz}$, the average residual stress is $-194 \mathrm{MPa}$, and when the reciprocating frequency increases to $150 \mathrm{~Hz}$, the average residual stress is $-309 \mathrm{MPa}$. So, the residual compressive stress increases with the increase of the reciprocating frequency. At the same time, with the increase of the reciprocating frequency, the consistency of the residual compressive stress is improved.

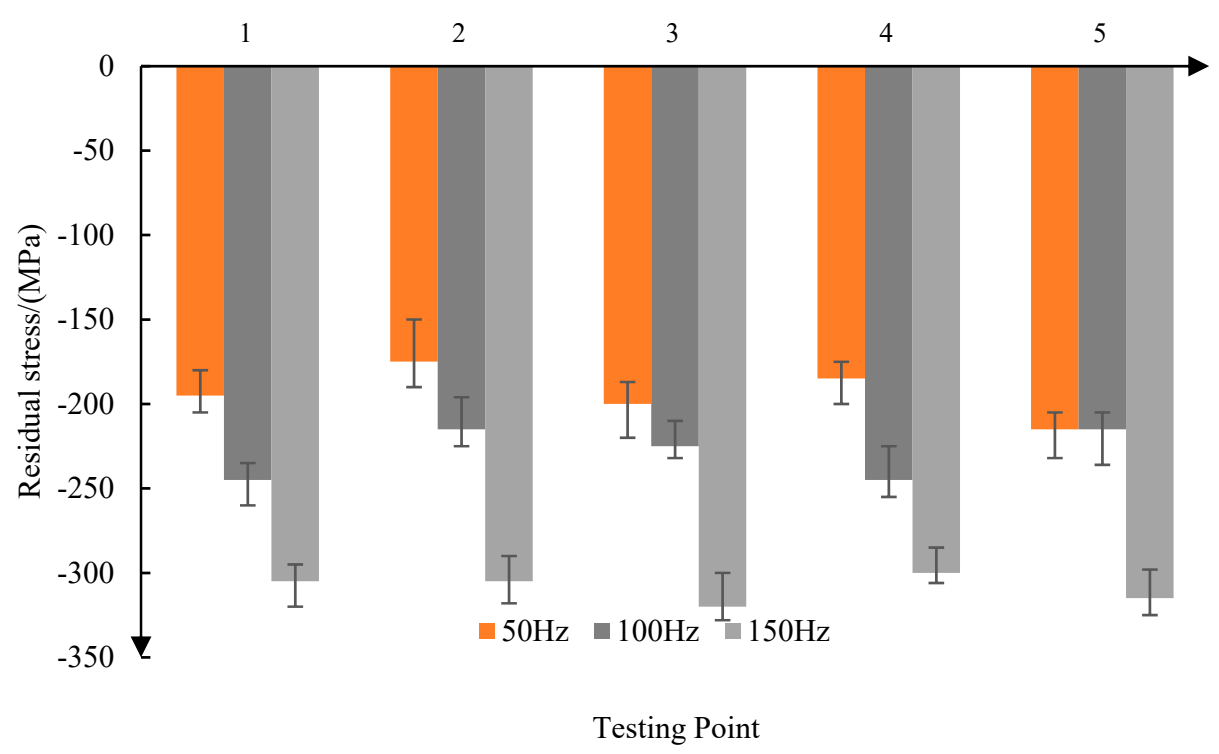

Figure 6. Effect of reciprocating frequency on residual stress.

As shown in Figure 7, when the feed rate is $0.5 \mathrm{~m} / \mathrm{min}$, the average residual stress is $-311 \mathrm{MPa}$. When the feed velocity increases to $1.5 \mathrm{~m} / \mathrm{min}$, the average residual stress is $-224 \mathrm{MPa}$. So, the residual compressive stress decreases with the increase of the feed rate. At the same time, with the decrease of the feed speed, the consistency of the residual compressive stress is improved.

From the above analysis, it can be seen that the residual compressive stress produced by abrasive belt grinding is in the range of 120-300 MPa. With the increase of grinding contact force, the increase of reciprocating frequency, and the decrease of feed speed, the residual compressive stress on the surface of the parts increases. 


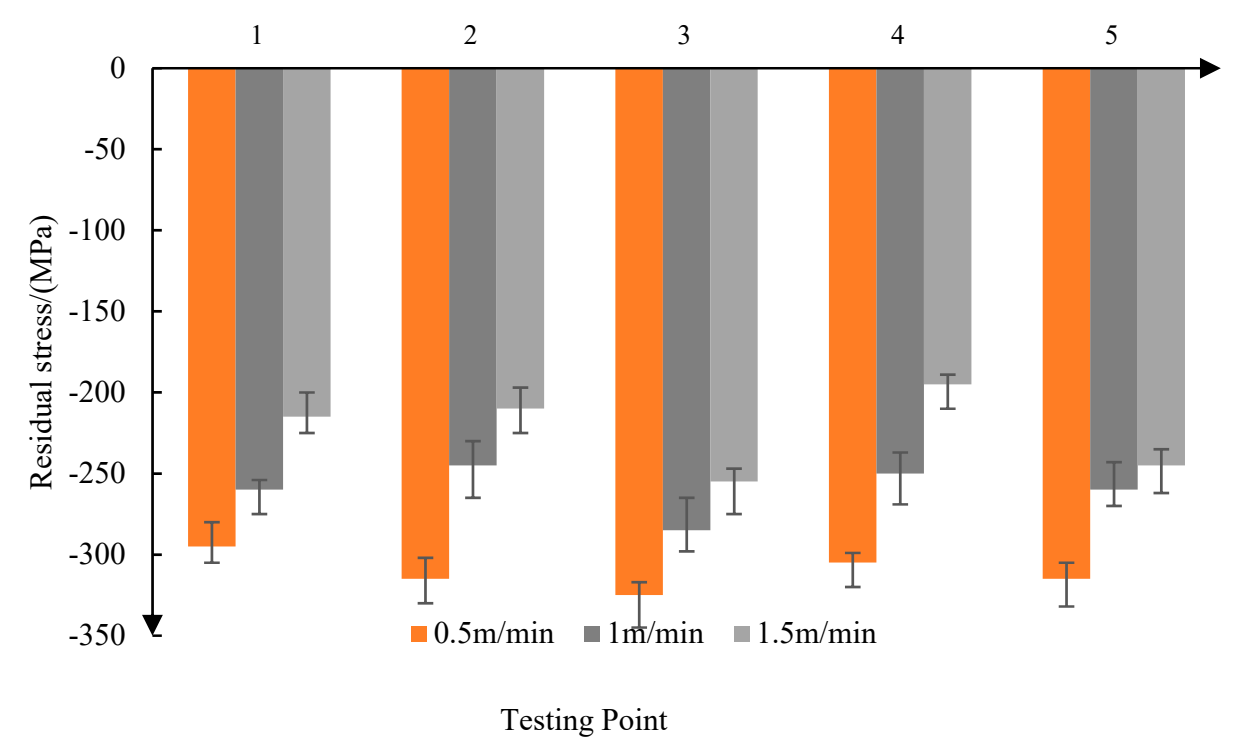

Figure 7. Effect of feed rate on residual stress.

\section{Analysis of Surface Residual Stress Characteristics}

\subsection{Finite Element Analysis}

Because this model is regular and simple in shape, the sweep method is used to divide it. The meshes are hexahedron, the middle meshes are fine, and the ends are wide, so that the meshes and the fatigue life of the middle sections can be calculated more quickly and accurately, as the middle is the position to analyse. The details of the element division are shown in Figure 8.

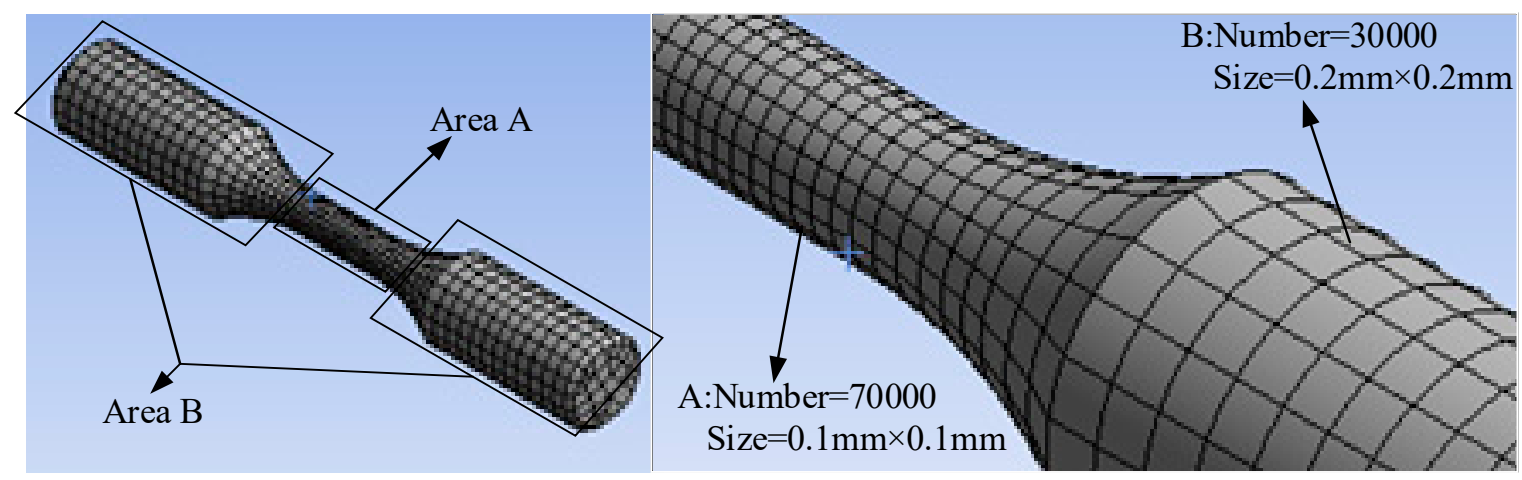

Figure 8. Element division.

When the tensile force is applied to the middle surface of the bar, the stress value of the intermediate surface increases significantly under the pressure, which indicates that the tensile stress of the middle surface is superimposed with the load applied at both ends, thus increasing the stress value of the intermediate surface. This is because the applied residual stress can neutralize a part of the load acting on the intermediate surface to a certain extent. By applying the normal tensile stress, the simulation residual stress values of the surface are found to be $110 \mathrm{MPa}, 220 \mathrm{MPa}$, and $310 \mathrm{MPa}$, respectively, as shown in Figure 9. 


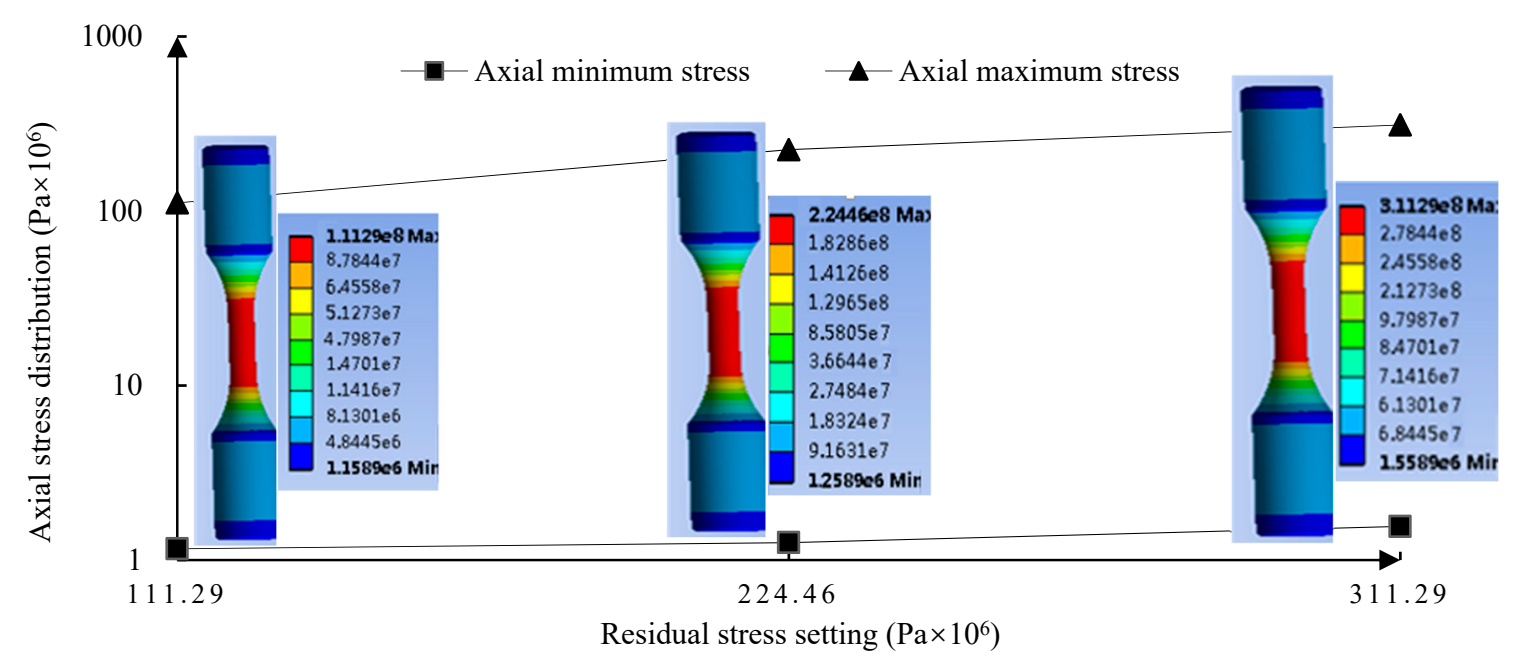

Figure 9. Stress distribution after normal stress is applied.

\subsection{Analysis of Fatigue Life}

(1) The ANSYS Workbench software material library provides the common fatigue data of iron, magnesium alloys, titanium alloys, and so on, mainly including the general data information of the material, the fatigue life curve of high and low cycles, and so on. The properties of the simulation materials are set according to Tables 1 and 2 . The $\mathrm{S}-\mathrm{N}$ curves (the fatigue strength of the material standard specimen is the ordinate, and the logarithmic value $\lg \mathrm{N}$ of the fatigue life is taken as the transverse coordinate; the curve of the relationship between the fatigue strength and the fatigue life of the standard specimen under certain cyclic characteristics is expressed) of TC17 are set according to the experimental data.

(2) The loading mode used in this experiment is the stress control, the cyclic stress spectrum of the sinusoidal pulse is used, and the cyclic characteristic stress ratio, $R$, is 0.1 , and the frequency is $50 \mathrm{~Hz}$. By changing the scale factor to enlarge and reduce the force of the static analysis in front, we can obtain the desired stress values, and thus carry out the fatigue simulation experiment under different stress levels.

(3) Figure 10 shows the fatigue life curves of titanium alloy bars under different residual stresses under varying grinding process parameters. The process parameters are as follows: the contact force is $10 \mathrm{~N}, 20 \mathrm{~N}$, and $30 \mathrm{~N}$; the reciprocating frequency is $50 \mathrm{~Hz}, 100 \mathrm{~Hz}$, and $150 \mathrm{~Hz}$; and the feed speed is $0.5 \mathrm{~m} / \mathrm{min}, 1.0 \mathrm{~m} / \mathrm{min}$, and $1.5 \mathrm{~m} / \mathrm{min}$, respectively.

As can be seen from Figure 10a, with the increase of the grinding contact force, $F_{\mathrm{a}}$, the fatigue life of $550 \mathrm{MPa}$ increases from $9.97 \times 10^{6}$ cycles to $5.5 \times 10^{7}$ cycles when the reciprocating frequency, $f$, is $50 \mathrm{~Hz}$ and the feed speed $v_{\mathrm{p}}$ is $1.5 \mathrm{~m} / \mathrm{min}$. Figure $10 \mathrm{~b}$ shows that when the contact force, $F_{\mathrm{a}}$, is $10 \mathrm{~N}$ and the feed velocity, $v_{\mathrm{p}}$, is $1.5 \mathrm{~m} / \mathrm{min}$, the fatigue life increases from $9.97 \times 10^{6}$ cycles to $3.32 \times 10^{7}$ cycles. With the increase of the grinding reciprocating frequency, the fatigue life increases from $9.97 \times 10^{6}$ cycles to $3.32 \times 10^{7}$ cycles under the tensile loading level of $550 \mathrm{MPa}$, and it can be seen from Figure $10 \mathrm{c}$ that when the contact force, $F_{\mathrm{a}}$, is $10 \mathrm{~N}$ and the reciprocating frequency, $f$, is $50 \mathrm{~Hz}$, the fatigue life decreases with the decrease of the feed speed. The fatigue life increased from $9.97 \times 10^{6}$ cycles to $3.98 \times 10^{7}$ cycles under the tensile load level of $550 \mathrm{MPa}$. It can be seen from Figure 10d that the greater the residual compressive stress, the greater the fatigue life at the same load stress level. The curve is vertical at $1 \times 10^{8}$ cycles, which is attributable the maximum fatigue life of $1 \times 10^{8}$ cycles when the S-N curve of TC17 is set in the material library, so the software takes $1 \times 10^{8}$ cycles as the limit fatigue value in the analysis. 


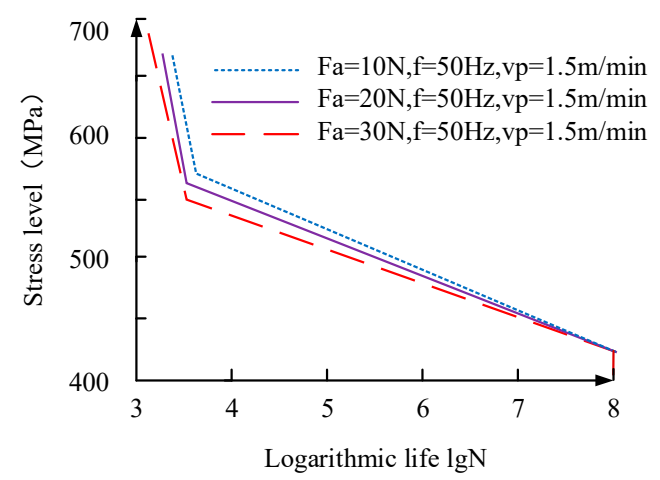

(a) Fatigue life curves under different contact forces

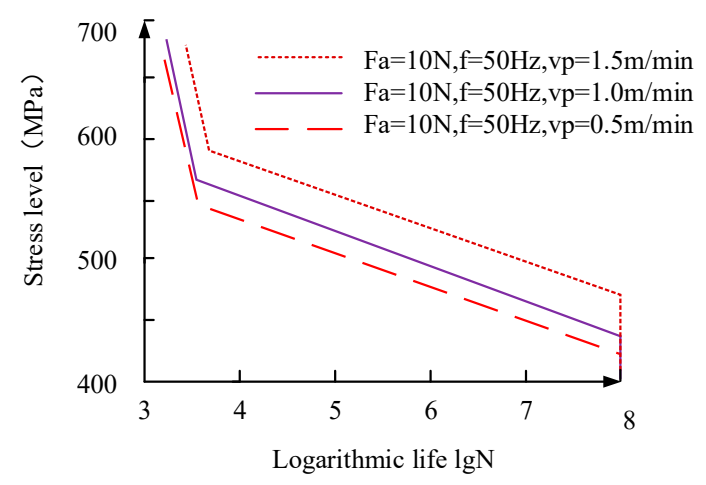

(c) Fatigue life curves under different feed speed

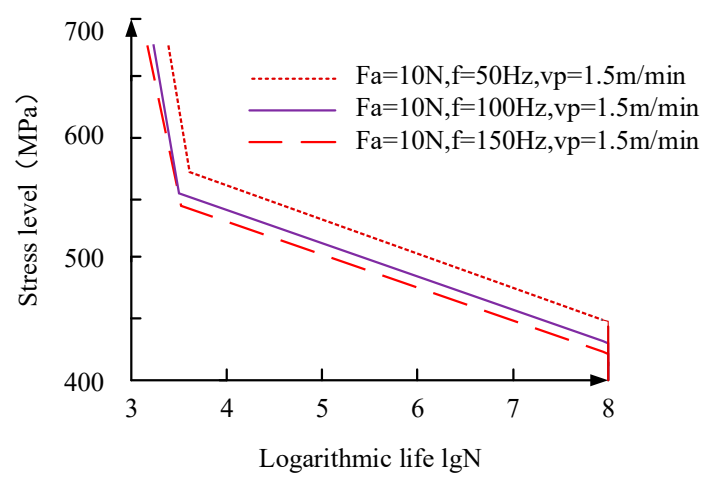

(b) Fatigue life curves under different reciprocating frequency

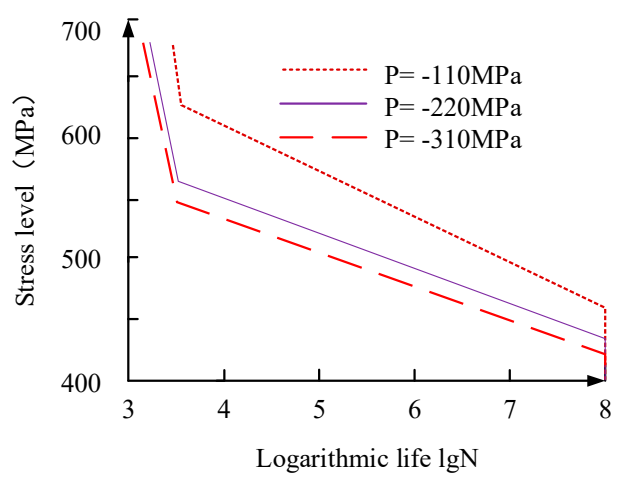

(d) Fatigue life under different residual stress

Figure 10. Fatigue life curves: (a) under different forces on the surface; (b) under a different reciprocating frequency; (c) under different feed speed; (d) under different residual stress.

It can also be seen from Figure 10 that when the working stress is about $550 \mathrm{MPa}$, the slope of the fatigue life curve changes, and with the increase of residual compressive stress, the point of slope change of the fatigue curve moves upward, relative to the axis. The reason for this is that when the work stress increases, the fatigue crack initiation on the surface has a more obvious stress concentration; so, it has less fatigue strength and tends to have a bigger slope, which causes less cycles. When the surface compressive residual stress increases, the crack initiation appears from the surface to the subsurface, where it tends to have less ability to take the place of the stress concentration; thus, at the same stress level, it can bear more load cycles.

In general, the residual stress is mainly divided into two types, residual compressive stress and residual tensile stress. In general, the so-called residual stress is called residual tensile stress, which exists as an outward pressure on the surface of the workpiece. This is an undesirable force that plays a significant role in the deformation of the workpiece, the initiation of cracks, the cracking, and surface wear; and the residual compressive stress is a desirable residual stress, which is the opposite to the tensile stress. It exists on the surface of the workpiece as an inward pressure, rather than as an outward tension. Therefore, if there is residual compressive stress on the surface of the workpiece, the fatigue strength and fatigue life of the workpiece will be greatly improved, and the wear resistance and corrosion resistance will be much better [31].

\subsection{Fatigue Test Analysis}

\subsubsection{Fatigue Test}

(1) Fatigue Test Equipment and Parameters

The experiment was carried out on a MTS809-10T tension torsion static fatigue test system (MTS809-10T, MTS, Eden Prairie, MN, USA). The system is mainly composed of an 809 hydraulic servo 
material test bench, a tension and torsion compound load sensor, a side load static actuator, and a MTS TESTSTAR digital control system. The main technical indicators are as follows: maximum dynamic pull, $100 \mathrm{kN}$, and torque, $1000 \mathrm{NM}$. The size of the standard specimen is as follows: the diameter of the round rod specimen is $6-30 \mathrm{~mm}$, and the thickness of the plate specimen is less than $12 \mathrm{~mm}$ and the width is less than $130 \mathrm{~mm}$. The load we used in this experiment was sinusoidal, with a frequency of $50 \mathrm{~Hz}$ and a cyclic characteristic stress ratio, $R$, of 0.1 . The value of the loads were $450 \mathrm{MPa}, 475 \mathrm{MPa}$, $500 \mathrm{MPa}$, and $525 \mathrm{MPa}$, respectively.

\section{(2) Fatigue Test Data}

The fatigue data obtained from the tensile tests on the specimens with different surface residual compressive stress (measured by X-ray) using the testing machine are shown in Table 3. From the eight groups of data, we can see that the measured experimental data are in line with the reality that a specimen that does not necessarily need to be removed because of defects in the material and processing process. Under the same working stress, the fatigue life increases with the increase of residual compressive stress, and the fatigue life decreases with the increase of working stress, under the same residual compressive stress.

Table 3. Fatigue life of specimens with different surface integrity and stress levels.

\begin{tabular}{ccccc}
\hline Sample & Residual Stress (MPa) & Working Stress (MPa) & Fatigue Life (Cycle) & Logarithmic Life (lg N) \\
\hline 1 & -284 & 450 & $11,324,681$ & 7.05402 \\
2 & -250 & 450 & $11,223,465$ & 7.05012 \\
3 & -214 & 475 & $9,876,522$ & 6.99460 \\
4 & -202 & 475 & $9,798,325$ & 6.99115 \\
5 & -287 & 500 & $9,628,951$ & 6.98357 \\
6 & -298 & 500 & $9,668,362$ & 6.98535 \\
7 & -147 & 525 & 542,957 & 5.80818 \\
8 & -150 & 525 & 547,132 & 5.73809 \\
\hline
\end{tabular}

\subsubsection{Simulation Experiment Data Analysis}

The data of the fatigue life from the simulation and experiment were compared and analyzed. As a whole, the error between the experimental data and our simulation results, as shown in Figure 11b, is less than $10 \%$, which further illustrates the appropriateness of the simulation.

It can be seen from Figure 11a that the fatigue life according to the simulation data is always higher than the actual experimental data, which is because, in addition to the effects of roughness, surface hardness, residual stress, and other stress levels, there are also various uncertainties in the experimental environment, such as temperature changes, the imprecise measurement of integrity parameters, and so on. The lifetimes of samples 7 and 8 were significantly lower than that of the other samples, because the working stress of these two samples' groups was the highest, and the compressive residual stress on the surface was the lowest. According to the previous simulation experiments, we can see that the increase of the surface compressive residual stress can increase the fatigue life, and the increase of the working stress at both ends can reduce the fatigue life. The comparison between samples 6 and 7 and samples 5 and 6 shows that the increase of working stress is $25 \mathrm{MPa}$, while the increase of residual stress is $80 \mathrm{MPa}$ on average, but the fatigue life between them has little difference. The fatigue life of samples 5 and 6 is about $2 \%$ higher than that of samples 6 and 7. It can be seen that in this experiment, the influence of working stress on fatigue life is greater than that of residual compressive stress. 


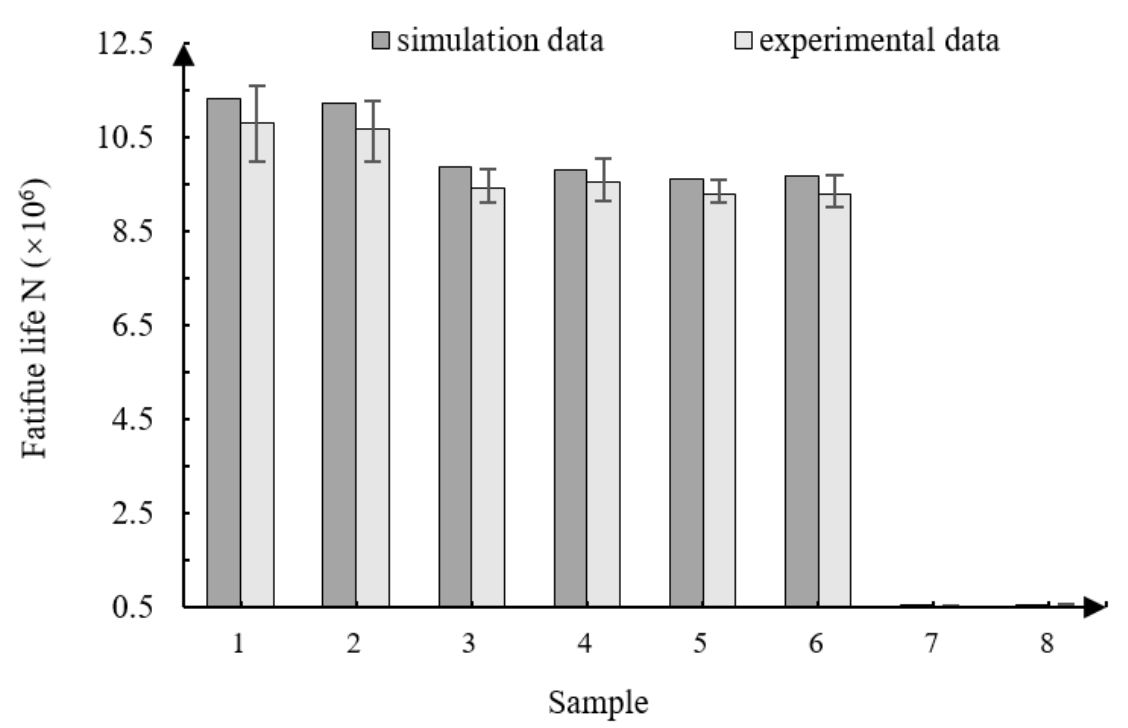

(a)

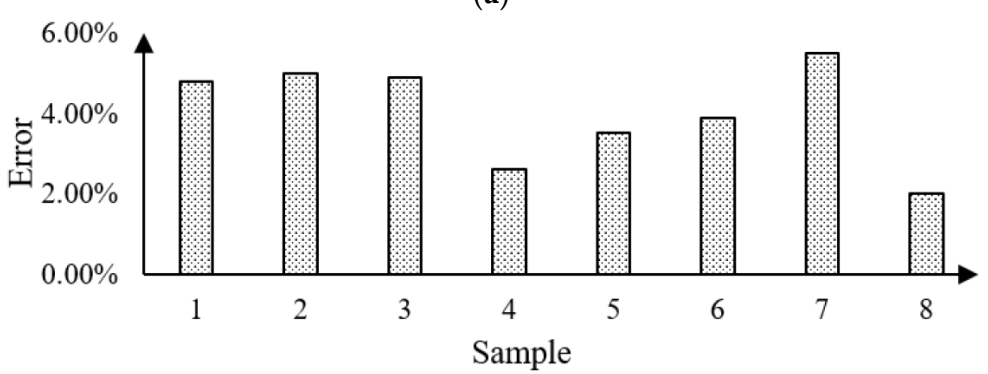

(b)

Figure 11. Simulation data and experimental data error. (a) Comparison of simulation data and experimental data; (b) error between simulation data and experimental data.

\subsubsection{Analysis of Fatigue Fractures}

The macroscopic morphology of a fatigue fracture is divided into the following three parts: the fatigue source (source), crack propagation (propagation), and final fracture (transient fracture). A typical tested bar is chosen for the analysis, and the residual stress is $300 \mathrm{MPa}$ under the working stress of $500 \mathrm{MPa}$. Figure 12 shows the microscopic figures of the final fracture zone. It can be clearly seen from Figure $12 \mathrm{~b}$ that the strength limit of the material is exceeded in the transient fault area, because the area under force is too small to cope. The plastic deformation fracture occurs in the middle part, and the shear port presents a $45^{\circ}$ angle and slip separation. In Figure 12c, the material is slip-fractured during the deformation in Figure 12b. The macroscopic fracture surface is smooth in Figure $12 \mathrm{~d}$. This is due to repeated extrusion friction at crack initiation under high cycle fatigue.

Figure 13 shows that under the same stress level and different residual compressive stress, the fatigue crack initiation point is different; the arrows indicate where the fatigue crack initiation occurs at the residual compressive stress level. It can be seen from Figure 13a that the fatigue crack initiation point is on the surface of the specimen under low residual compressive stress, and that the fatigue crack initiation occurs on the subsurface of the specimen under high residual pressure, as shown in Figure 13b. The results show that the larger the residual compressive stress is, the less the fatigue crack initiation occurs on the surface, and the better the surface quality is. Figure 14 shows the TC17 fatigue crack growth zone, from which we can see the typical fatigue bands (areas A, B, C, D, and E). Obvious secondary crack characteristics have been found. 

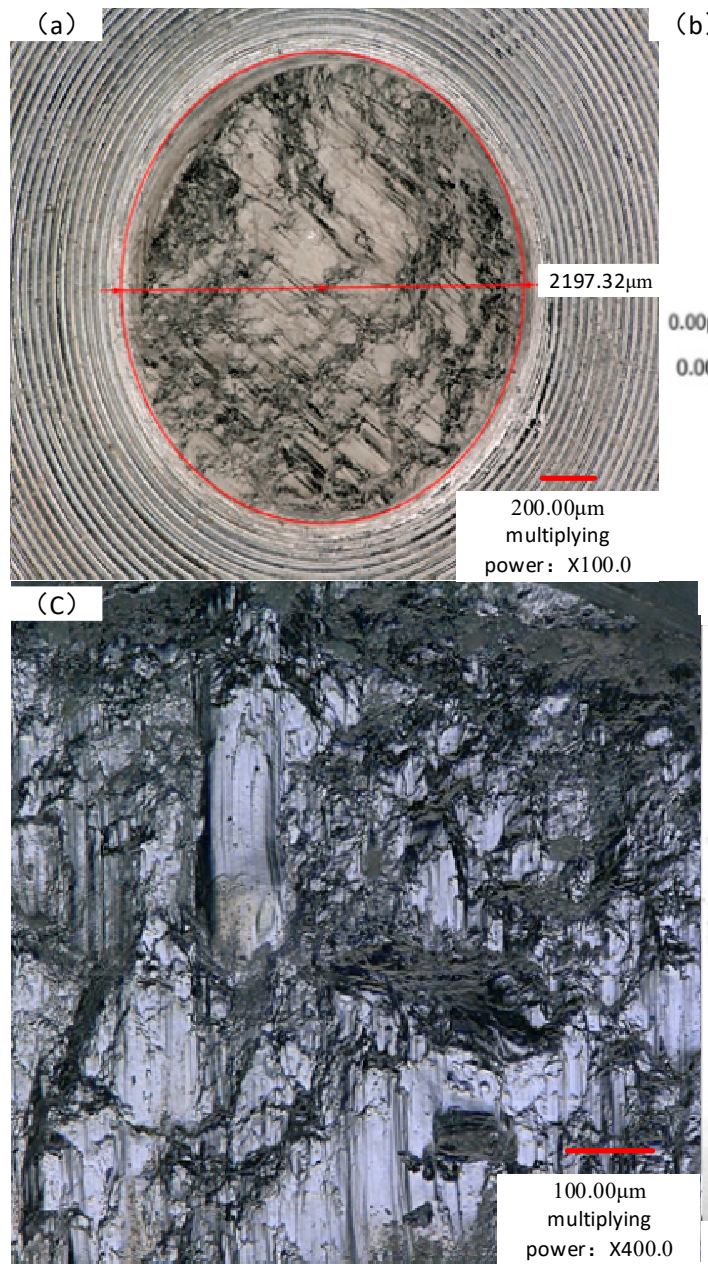

(b)

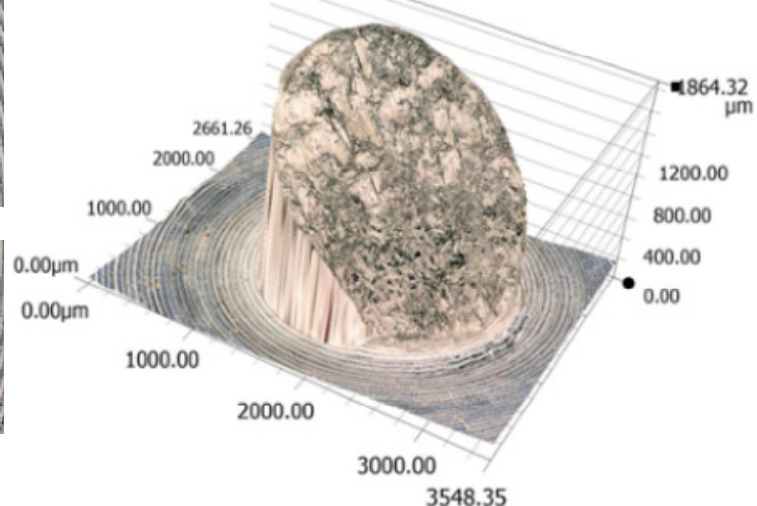

(d)

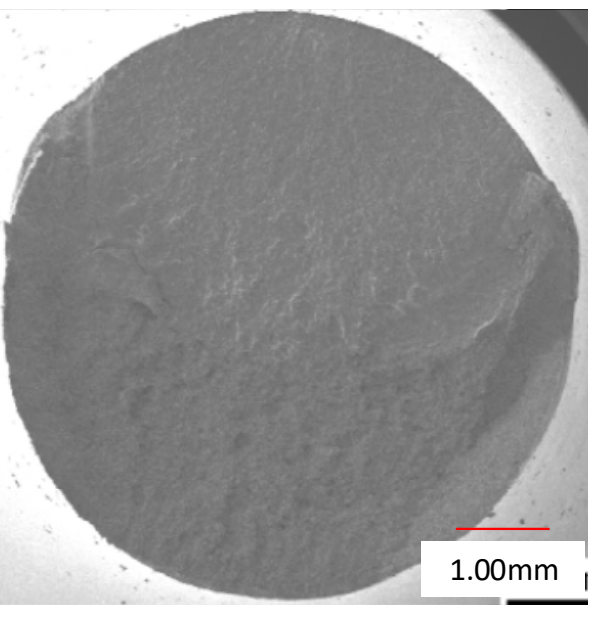

Figure 12. Final fracture area macro map: (a) radial microscopic observation; (b) microscopic size distribution around a plastic fracture; (c) macroscopic map of the final fault zone; (d) macroscopic fracture surface.

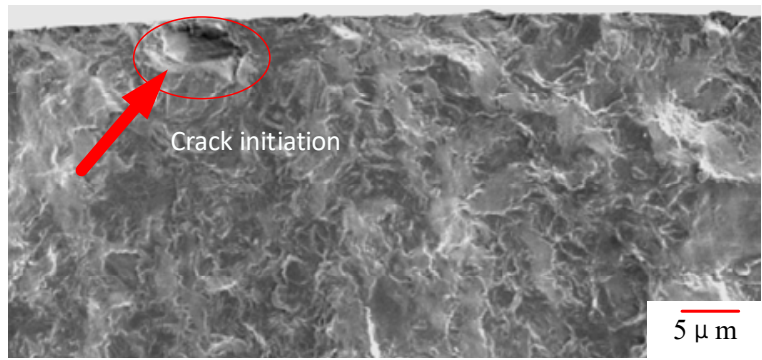

(a) Residual stress -150MPa

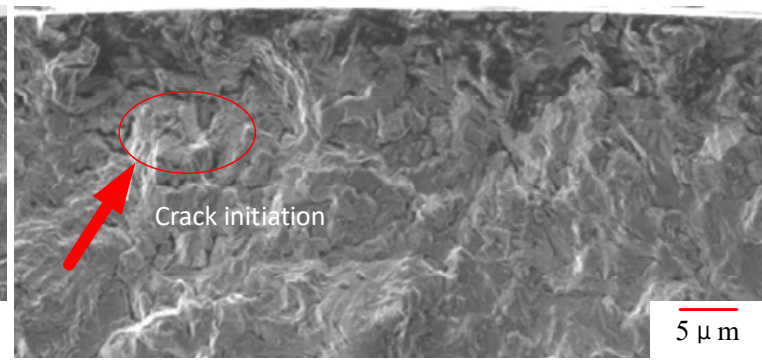

(b) Residual stress -500MPa

Figure 13. Fatigue crack initiation under different residual stresses in a cross-section view: (a) under the residual stress of $-550 \mathrm{MPa}$; (b) under the residual stress of $-500 \mathrm{MPa}$.

The port morphology of the fatigue specimen was observed using SEM, as shown in Figure 15. The center of the port exhibited a ductile fracture, which was characterized by typical uniaxial tensile equiaxed dimples. There were no obvious nucleation particles in the dimples, and they were all small equiaxed dimples. There are a few large dimples, and these dimples contain a few smaller dimples, and some dimples have tiny holes in them. The fracture morphology at room temperature is of a ductile fracture and fine equiaxed dimples. The dimples in Figure 15b are larger than those in Figure 15a. The bigger dimple size confers a good plasticity. The larger the residual stress is, the larger 
the dimple is and the better the surface is. So, the increase of residual stress can improve the plasticity to some extent.

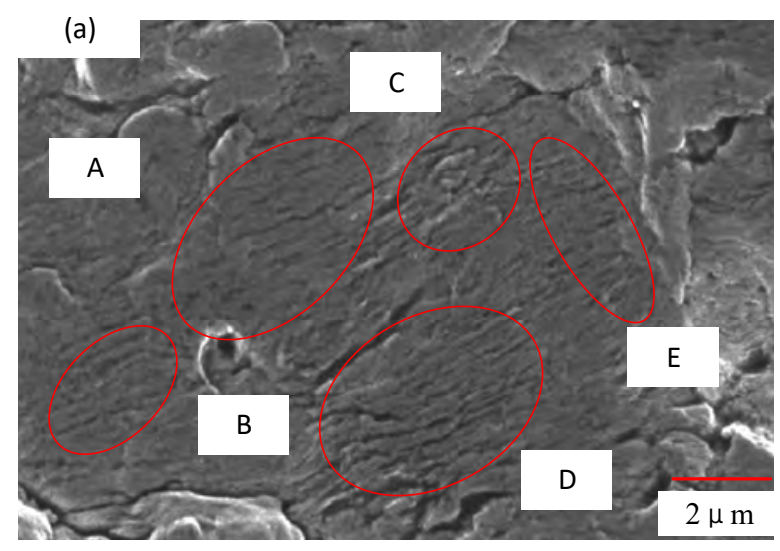

Figure 14. Fatigue crack growth.
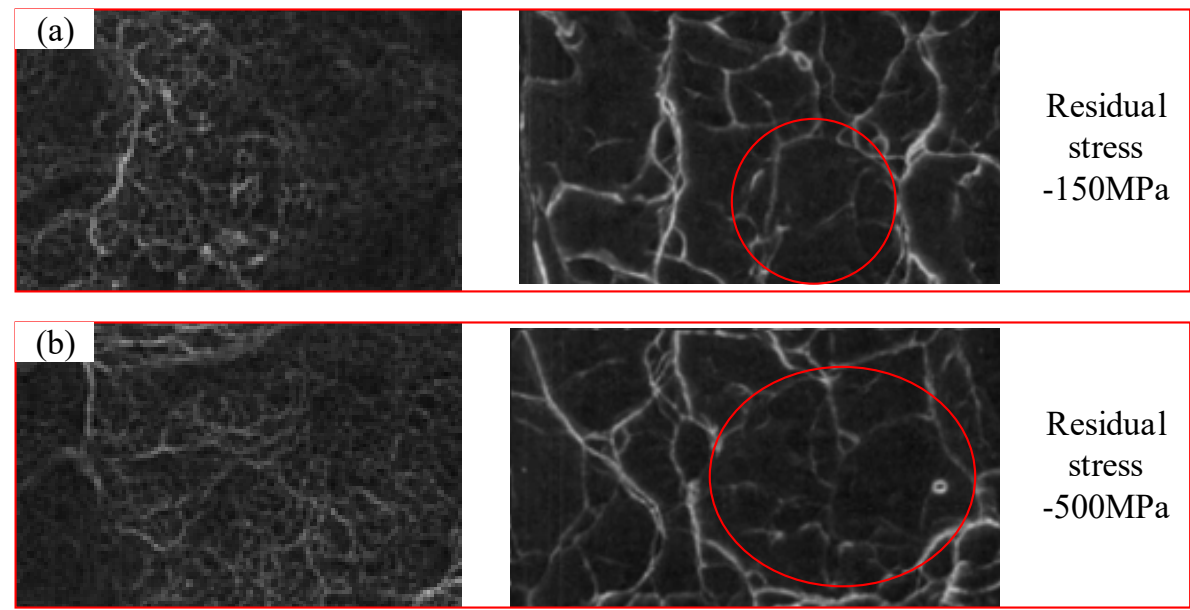

Figure 15. SEM fracture morphology: (a) under the residual stress of -150 MPa; (b) under the residual stress of $-500 \mathrm{MPa}$.

\section{Conclusions}

In this paper, the residual stress of a TC17 titanium alloy after belt grinding and its impact on the fatigue life were studied.

Firstly, the residual stress produced by grinding the titanium alloy surface was found to be between -120 and $-300 \mathrm{MPa}$. The residual compressive stress increases with the increase of depth when the surface depth is less than $1.2 \mu \mathrm{m}$, and when the surface depth layer is greater than $1.2 \mu \mathrm{m}$, the residual compressive stress decreases with the increase of the surface depth. The experimental results show that with the increase of the grinding contact force, the increase of the reciprocating frequency, and the decrease of the feed speed, the residual compressive stress on the surface of the parts increases, and the fatigue life is higher at the same working stress level. Based on Bragg's law, the surface residual stress is represented by normal tension and pressure on the surface of the bar in order to simulate the residual stress, and then the residual stress model is simulated using the tensile force on the surface of the model.

Then, the fatigue life of the bar subjected to a sinusoidal tensile load at both ends was analyzed by simulating the fatigue test of the titanium alloy bar. The fatigue life of the same working stress level increased with the increase of the grinding contact force. With the increase of the reciprocating frequency, the fatigue life of the same working stress level increased, and with the decrease of the feed speed, the fatigue life of the same working stress level was higher. 
The slope of the fatigue life curve changes at about $550 \mathrm{MPa}$, and with the increase of residual compressive stress, the point of slope change of the fatigue curve moves upward relative to the axis. The reason for this is that when the working stress increases, the fatigue crack initiation on the surface has a more obvious stress concentration, and thus has a lower fatigue strength and tends to have a bigger slope, which means fewer cycles to fatigue. When the surface compressive residual increases, the location where the crack initiation begins shifts from the surface to the subsurface, where it tends to have less ability to take the place of the stress concentration; thus, at the same stress level, it can bear more load cycles.

Finally, fatigue experiments were carried out and the fracture surface was analyzed. The error between the simulation data and the experimental data was less than $10 \%$, and the fracture morphology at room temperature was of a ductile fracture and fine equiaxed dimples. The greater the residual compressive stress is, the better the surface quality and the less apparent the fatigue crack initiation point is on the surface. From the propagation, we can see the typical fatigue bands and obvious secondary crack characteristics.

Author Contributions: Conceptualization, Y.H. (Yi He) and G.X.; Methodology, G.X.; Software Y.H. (Yi He); Validation, Y.H. (Yi He), G.X., W.L. and Y.H. (Yun Huang); Formal Analysis, Y.H. (Yi He), G.X. and W.L.; Investigation, Y.H. (Yi He) and Y.H. (Yun Huang); Resources, G.X.; Data Curation, G.X. and W.L.; Writing-Original Draft Preparation, Y.H. (Yi He) and G.X.; Writing-Review \& Editing, G.X. and Y.H. (Yun Huang); Visualization, G.X. and W.L.; Supervision, G.X.; Project Administration, G.X.; Funding Acquisition, G.X. and Y.H. (Yun Huang).

Funding: This work was supported by the National Natural Science Foundation of China (grant no. 51705047), the Technological Innovation and Application Demonstration of Chongqing (cstc2018jszx-cyzdX0061), the Fundamental Research Funds for the Central Universities (2018CDQYCD0038), and the major projects of aero engines and gas turbines (2-2017-VII-0002).

Conflicts of Interest: The authors declare no conflict of interest.

\section{References}

1. Brotzu, A.; Felli, F.; Marra, F; Pilone, D.; Pulci, G. Mechanical properties of a TiAl-based alloy at room and high temperatures. Mater. Sci. Technol. 2018, 34, 1847-1853. [CrossRef]

2. Zhu, S.P.; Yue, P.; Yu, Z.Y.; Wang, Q.Y. A combined high and low cycle fatigue model for life prediction of turbine blades. Materials 2017, 10, 698. [CrossRef] [PubMed]

3. Wang, H.; Wang, L.W.; Wang, T. Reconstruction and repair method of aircraft engine damage blade. Acta Aeronaut. Astronaut. Sin. 2016, 37, 1036-1048.

4. Bhaumik, S.K.; Sujata, M.; Venkataswamy, M.A. Fatigue failure of aircraft components. Eng. Fail. Anal. 2008, 15, 675-694. [CrossRef]

5. Bigerelle, M.; Gautier, A.; Hagege, B.; Favergeon, J.; Bounichane, B. Roughness characteristic length scales of belt finished surface. J. Mater. Process. Technol. 2009, 209, 6103-6116. [CrossRef]

6. Bigerelle, M.; Gautier, A.; Hagege, B. Mechanical modelling of micro-scale abrasion in superfinish belt grinding. Tribol. Int. 2008, 41, 992-1001. [CrossRef]

7. Jourani, A.; Dursapt, M.; Hamdi, H.; Rech, J.; Zahouani, H. Effect of the belt grinding on the surface texture: modeling of the contact and abrasive wear. Wear 2005, 259, 1137-1143. [CrossRef]

8. Segreto, T.; Karam, S.; Teti, R. Signal processing and pattern recognition for surface roughness assessment in multiple sensor monitoring of robot-assisted polishing. Int. J. Adv. Manuf. Technol. 2017, 90, 1023-1033. [CrossRef]

9. Eriksen, R.S.; Arentoft, M.; Gronbak, J.; Bay, N. Manufacture of functional surfaces through combined application of tool manufacturing processes and robot assisted polishing. CIRP Ann. Manuf. Technol. 2012, 61, 563-566. [CrossRef]

10. Xiao, G.J.; Huang, Y. Constant-load adaptive belt polishing of the weak-rigidity blisk blade. Int. J. Adv. Manuf. Technol. 2015, 78, 1473-1484. [CrossRef]

11. Zhao, T.; Shi, Y.Y.; Lin, X.J.; Duan, J.H.; Sun, P.C.; Zhang, J. Surface roughness prediction and parameters optimization in grinding and polishing process for IBR of aero-engine. Int. J. Adv. Manuf. Technol. 2014, 74, 653-663. [CrossRef] 
12. Xiao, G.J.; Huang, Y. Adaptive belt precision grinding for the weak rigidity deformation of blisk leading and trailing edge. Adv. Mech. Eng. 2017, 9, 1-12. [CrossRef]

13. Huang, Y.; Xiao, G.J.; Zhao, H.Q.; Zou, L.; Zhao, L.; Liu, Y.; Dai, W.T. Residual stress of belt polishing for the micro-stiffener surface on the titanium alloys. Procedia CIRP 2018, 71, 11-15. [CrossRef]

14. Dong, Z.G.; Cao, K.; Kang, R.K.; Hao, B.J.; Zhen, F.F. Experimental research on grinding titanium alloy TC17 with microcrystalline corundum grinding wheel. Diam. Abrasives Eng. 2015, 35, 14-20.

15. Jeelani, S.; Ramakrishnan, K. Subsurface plastic deformation in machining 6A1-2Sn-4Zr-2Mo titanium alloy. Wear 1983, 85, 121-130. [CrossRef]

16. Mantle, A.L.; Aspinwall, D.K. Surface integrity of a high speed milled gamma titanium aluminide. J. Mater. Process Technol. 2001, 118, 143-150. [CrossRef]

17. Lee, S.; Kim, T.; Chou, Y. The effect of surface roughness on contact fatigue behavior using mesoscopic approach. Tribol. Lett. 2009, 36, 269-276. [CrossRef]

18. Bhaumil, S.K.; Bhaskaran, T.A.; Rangaraju, R.; Venkataswamy, M.A.; Parameswara, M.A.; Krishnan, R.V. Failure of turbine rotor blisk of an aircraft engine. Eng. Fail. Anal. 2002, 9, 287-301. [CrossRef]

19. James, M.N.; Newby, M.; Hattingh, G.; Steuwer, A. Shot-Peening of steam turbine blades residual stresses and their modification by fatigue cycling. Procedia Eng. 2010, 2, 441-451. [CrossRef]

20. Huang, J.; Ren, J.X.; Zhang, J.C. Effect of surface integrity on high and low cycle fatigue life of GH33A. Acta Aeronaut. Astronaut. Sin. 1991, 10, 528-531.

21. Sun, C.; Huang, Z.W. Effects of varied surface conditions on the fatigue behavior of a high-strength gamma-based titanium aluminide alloy. Rare Mental Mater. Eng. 2014, 43, 589-594.

22. Li, K.; Fu, X.S.; Li, Z.G.; Zhou, W.L.; Chen, G.Q. Fatigue fracture mechanism of Ti-6Al-4V alloy strengthened by wet peening treatment. Rare Mental Mater. Eng. 2017, 375, 3068-3072.

23. Wang, X.; Hu, Y.H.; Wang, X.; Tang, Z.; Zou, J. Effect of shot peening on fatigue performance stress-concentration sensitivity of FGH96 powder metallurgy superalloy. Aeronaut. Manuf. Technol. 2017, 532, $48-53$.

24. Zhou, L.C. The mechanism of laser shock composite reinforcement and its application in aero-engine turbine blades. J. Mech. Eng. 2016, 52, 201.

25. Wang, B.L. Study on Mechanical Properties and Machining Characteristics of Titanium Alloy TC17. Ph.D. Thesis, Shandong University, Jinan, China, 2013.

26. Yao, C.F.; Tan, L.; Yang, P.; Zhang, D.H. Effects of tool orientation and surface curvature on surface integrity in ball end milling of TC17. Int. J. Adv. Manuf. Technol. 2018, 94, 1699-1710. [CrossRef]

27. Moussaoui, K.; Segonds, S.; Rubio, W.; Mousseigne, M. Studying the measurement by X-ray diffraction of residual stresses in Ti6Al4V titanium alloy. Mater. Sci. Eng. A 2016, 667, 340-348. [CrossRef]

28. Friák, M.; Counts, W.A.; Ma, D.C.; Sander, B.; Holec, D.; Rabbe, D.; Neugebauer, J. Theory-guided materials design of multi-phase Ti-Nb alloys with bone-matching elastic properties. Materials 2012, 5, 1853-1872. [CrossRef]

29. Palkowski, H.; Brück, S.; Pirling, T.; Carradò, A. Investigation on the residual stress state of drawn tubes by numerical simulation and neutron diffraction analysis. Materials 2013, 6, 5118-5130. [CrossRef] [PubMed]

30. Wu, D.Q.; Cao, W.L.; Jing, H.Y.; Xu, L.Y.; Zhao, L.; Han, Y.D. Analytical and numerical investigations of creep crack initiation considering the load-independent constraint parameter Q. Arch. Appl. Mech. 2018, 88, 2031-2050. [CrossRef]

31. Du, D.X.; Liu, D.X.; Zhang, X.H.; Tang, J.G.; Meng, B.L. Effect of WC-17Co coating combined with shot peening treatment on fatigue behaviors of TC21 titanium alloy. Materials 2016, 9, 865. [CrossRef] [PubMed]

(C) 2018 by the authors. Licensee MDPI, Basel, Switzerland. This article is an open access article distributed under the terms and conditions of the Creative Commons Attribution (CC BY) license (http://creativecommons.org/licenses/by/4.0/). 